\title{
Offline drone instrumentalized ambulance for emergency situations
}

\author{
Hitesh Mohapatra \\ Department of Computer Science and Engineering, Koneru Lakshmaiah Education Foundation, India \\ Department of Computer Science and Engineering, VSS University, India
}

\section{Article Info \\ Article history: \\ Received May 5, 2020 \\ Revised Jun 7, 2020 \\ Accepted Aug 4, 2020}

\section{Keywords:}

Accidents

Ambulance

Drone

Emergency

Medical help

\begin{abstract}
In this paper, an offline drone instrumentalized ambulance (ODIA) mechanism has been discussed. The rapid increase in the urban population directly influences every sector of society. The sectors are maybe food, health care, education, transportation, etc. Normally, it has been observed that when any accidents happen on the urban road or any remote places then, the availability of immediate medical help is very rare. It is not because of the unaware or unavailability of medical facilities rather it happens because of overcrowding on the urban road and geographical odd isolation of places. Hence, here an ODIA concept has been discussed which uses offline maps and offline first-aid medical videos through which immediate medical help can be made available at the patient end. This model helps to save the life of an accident victim by providing immediate medical attention. The key strength of ODIA is, it is independent of internet service that is why it is more suitable for harsh and hostile environments.
\end{abstract}

This is an open access article under the CC BY-SA license.

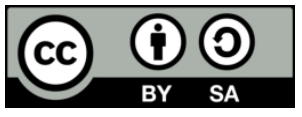

\section{Corresponding Author:}

Hitesh Mohapatra,

Department of Computer Science and Engineering,

Veer Surendra Sai University of Technology,

Burla, Sambalpur, Odisha, India, 768018.

Email: hiteshmahapatra@gmail.com

\section{INTRODUCTION}

The ambulance is a vehicle that is medically equipped to carry the patient from the source point to the hospitals. Sometimes, the outdoor patient also gets treated through the ambulance services. In 1487, for the first time, the ambulance has been used as an emergency service in Spain [1]. The rapid shifting of the rural population towards the urban area is an alarming situation for the smart city planner and designer [2]. It has been predicted that by $2030,70 \%$ of the total population will prefer to stay in urban places for better accessibility of the goods and services [3]. The gradual increase in the density level of urbanites directly influences several sectors of society such as food, education, health care, transportation, etc. In transportation, it can be easily observed the multiplied growth of vehicles on urban roads [4]. This volcanic growth of vehicles creates many major problems such as air pollution, traffic congestion, noise pollution, etc. In this crowd, if any accident happens then it is merely possible, that the ambulance will reach on time to help the accident victim. Though the ambulance departs on time from the hospitals but due to overcrowding on the road it is very difficult to reach on time at the required destination. As a consequence, many lives get lost. Hence, in this paper, a drone-based ambulance system has been proposed to reach the target point with minimum medical supplies. This can help to drag the lifetime of the victim until the ambulance arrived. Figure 1 illustrates the prototype of a drone model to carry the medical box for the source to the target point. The drone tracks emergency calls and uses GPS to navigate to the emergency location. The drone ambulance 
is the integration of three components such as live-stream webcam, first-aid box, and global positioning system (GPS) unit. Figure 1 illustrates the model of unmanned aerial vehicles (UAV) or drone for an offline drone instrumentalized ambulance (ODIA).

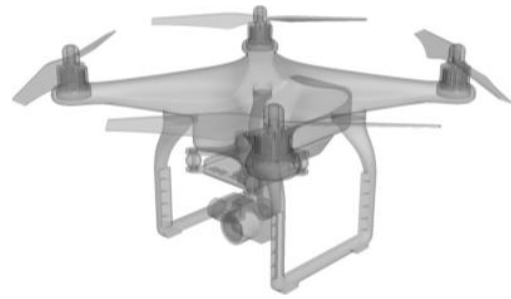

Figure 1. DJI Phantom 3 drone 3D CAD model for AutoCAD

\section{LITERATURE REVIEW}

Nearly a million Indians suffer from accidents each year, and only $8 \%$ will survive. This mainly happens because most of the time ambulances will not be able to reach on time due to heavy traffic on the road. So, by avoiding the traffic of the road the drone can fly up to the target point through which immediate medical attention can be provided to the victim. In this line of thought, few examples are available for showcase such as drone ambulance for cardiac arrest [5]. The drone or UAV can play a significant role in the various remote applications such as military, agriculture, product delivery, and medical [6]. Air transport is more efficient than ground transport as it doesn't face ground level restrictions. The primary benefit of air transport is it can reach to the inaccessible zones. As every coin has two sides, the air transport also has few constraints such as it is dependent upon weather conditions, it is compatible with low load transmission and it is highly costly. Hence, drone-based transportation cannot be affordable for daily uses whereas it can be used at the time of emergency.

The use of the drone for civil applications has started a new medium of transportation [7]. The transportation of goods, blood for the patient, capturing of images of uneven places (ex. Covid-19 tracking), etc. are made possible through drone $[8,9]$. Nowadays, the drone is available with a cost of $10-15 \mathrm{~K}$ that can fly up to 5-7 km. The growing use of drones in several domains brings many restrictions for the operating of a drone. These rules mostly associate with the weight of the drone. Based on the weight the drone owner must have a license to fly the drone. The first-ever civilian use of a drone can be viewed at "Haiti" to gauge the seriousness of an earthquake [10]. Another example of drone use is the Palo Festival in Nyon in 2012 [9]. Nowadays, the use of the drone is very much common in many events mostly at the grand parties and events. The mass production of drones makes able to bring the price to an affordable state. The UAV also helps in rescue operations, for example: 1) January of 2019 a search and rescue team in Snowy Canyon State Park, Utah, 2) January, 88-year-old Luis Reyna Zuniga was reported missing after leaving his home in Brownsville, Texas, 3) April of 2018, the Dalvik Search \& Rescue Team used a drone to find two cousins who were stranded on the side of a steep mountain in Dalvik, Iceland, etc. [11].

As earlier discussed the UAV is used in many domains, one such example where for the first time the drugs have been transferred between the German island of Juist (North Sea) and Norddeich, a city located in the north of Germany [12]. In the medical domain, there is another example exists which needs to be discussed to understand the importance of UAV. The Karolinska Institute, Sweden, has deployed a defibrillator to UAV to monitor and to help heart attack patients of the rural belts [13, 14]. Another study published in [15], states that the automated external defibrillator (AED) based UAV are improving in solving out-of-hospital cardiac arrest cases. These all examples justify the role of UAV or drones in several emergency sectors [16-18].

\section{RESEARCH GAP}

Normally, the UAV or drone is built on the standard quadcopter, which is driven by GPS or pilot or combination of both. It is designed with carbon fibers to keep drone lightweight and for power and motion, an electric motor driven by battery backup is used. The drone which is explicitly designed for health care sectors occupied with an embedded webcam for live streaming of the current situation. Countries like India or any other Asian country which still falls under the list of developing countries are lacking with many resources which are primary ground for drone service. The primary ground for a drone is high bandwidth internet at the remote places. Normally, in India, if the mobile goes a few kilometers away from the town 
then getting high-speed internet is quite difficult. In such cases, live streaming with drone cameras is a big question and also risky for the victim. Secondly, the absence of the internet also may create path loss as the UAV is driven by GPS. Hence, in this paper, an offline drone ambulance has been proposed where the UAV works on offline maps (within a stipulated zone) and offline medical videos in the absence of a live-streaming process.

\section{PROPOSED MODEL}

The movement of smart cities has been invited to many consequences with advancements. So, with this advancement, there must be a parallel line to deal with these negative consequences or faults [19-22]. In this section, the proposed model has been explained with detailed insights. As earlier said the UAV devices are mostly works on GPA and live streaming of webcam. In the developing countries, the lack of internet service at every corner of the city may fail the objective of UAV ambulances. Secondly, the country Like India which stands second in population ranking in the world has serious traffic problems on the road. In such circumstances, if any accidents happen at the remote places like national highways, isolated roads, rural places, or somewhere in the mid of river or coastal belts then offline drone ambulance (ODIA) can improve the medical services as it adopts offline maps and carries offline medical first-aid videos. Figure 2 illustrates the proposed model.

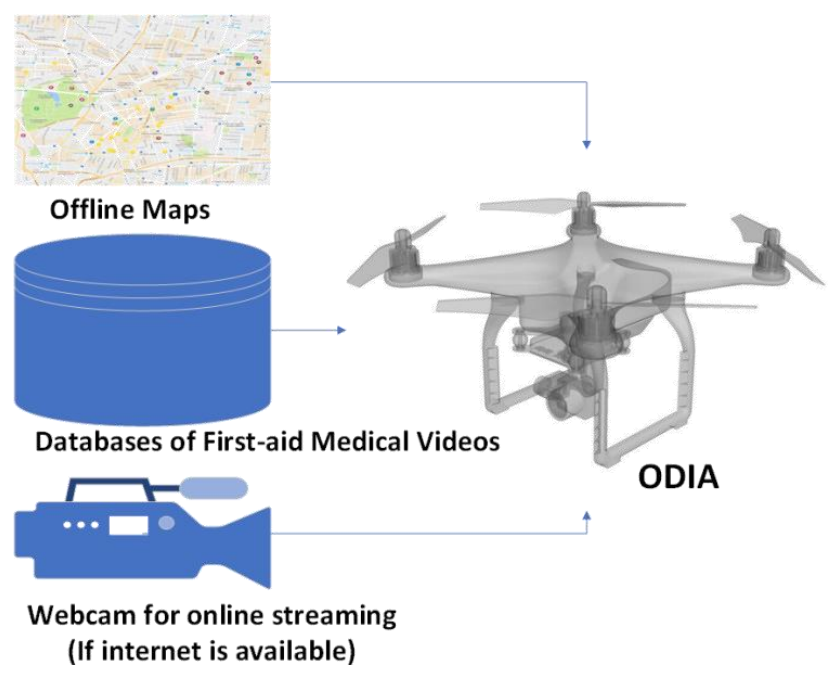

Figure 2. Offline drone ambulance

\subsection{ODIA with offline maps}

The UAVs are mostly GPS driven but there are many locations on the earth where there is no sign internet and GPS services. The available map services from Google, Apple, or Waze, these all are internet dependent. So, to enhance the services of ODIA the use of offline maps is more suggestible. Normally, the flying area of drone mostly stipulated within the range of $10-20 \mathrm{~km}$. The map of a $20 \mathrm{~km}$ radius can be downloaded from Google Maps and can be used for ODIA. Many applications are available from where the offline map can be downloaded. The built-in GPS radio can works smoothly on this offline map data and provides very close results in comparison to the maps-on-the-fly method [23]. This downloaded map can be active for 30 days upon expiring this map can be re-downloaded, or a local offline map can be loaded as a permanent basis. Figure 3 illustrates the screenshots of offline mapping.

\subsection{ODIA with pre-loaded first aid medical videos}

The live streaming with the patient is undoubtedly an efficient method to monitor the current health status of the patient or victim. However, the unavailability of the internet at all the places may lead to a critical situation through the drone has reached the target-spot on time. The nearby people of the victim may not be a medical doctor who can work by availing the first aids from the drone. Hence, in the case of ODIA, a visual unit has been provided which displays or plays the pre-loaded first-aid medical videos. The selection of these videos is done under the guidance of medical experts for emergencies. The availability of offline medical first aid videos can train the nearby-one to act as a doctor for the victim. At the time of need, these 
small videos sessions can save the life of a living being. Figure 4 [24] illustrates the drone with a display unit where the first-aid medical videos can be played. In case of unavailability of internet or online video streaming, through these videos, a layman can act as a doctor and can save the life of living being or at least can drag the life span for a few more times. In the mean while other tries can be done to reach the hospital.
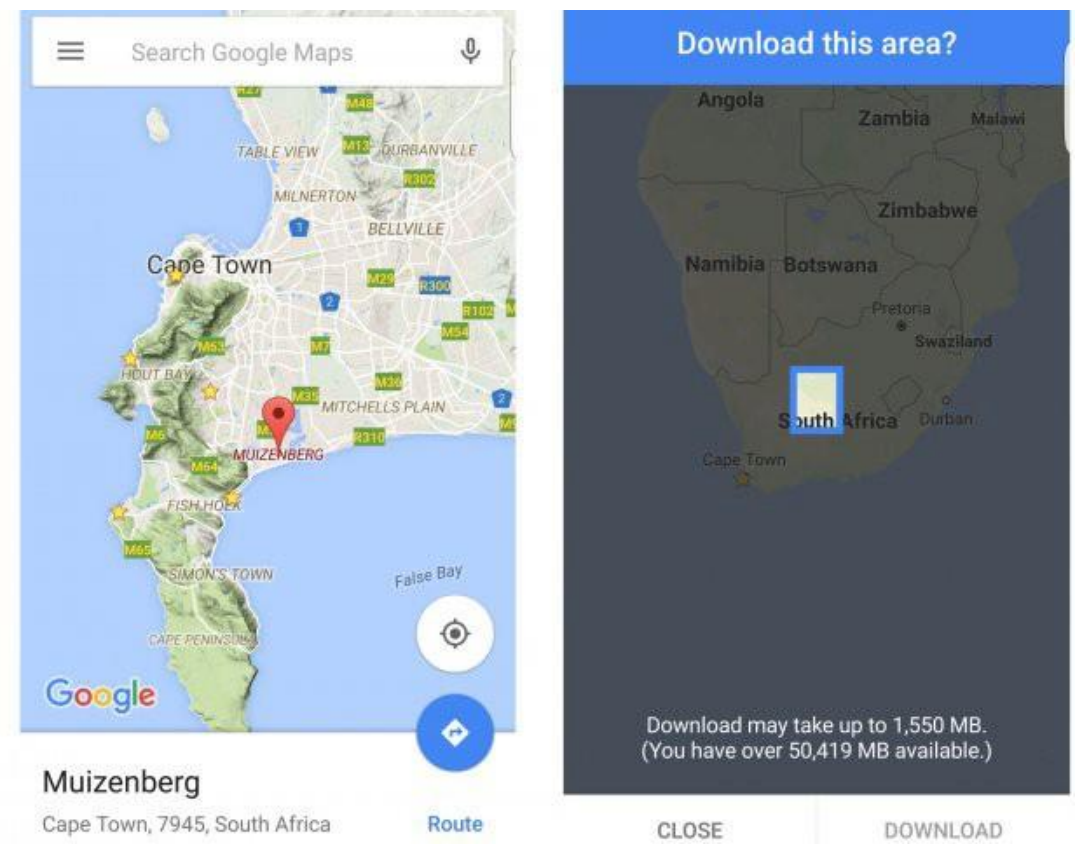

Figure 3. Offline map [23]

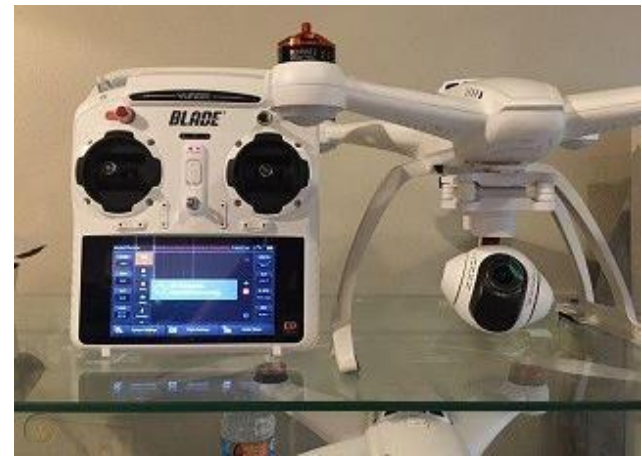

Figure 4. Drone with local display unit [24]

\section{CONCLUSION}

The volcanic growth of population and rapid urbanization becomes the biggest challenge for smart city planning. The various sectors of society such as education, transportation, health care, etc. are dependent upon these two core factors such as urbanization rate and population growth rate. The growth of the vehicle population on the urban road brings many problems for efficient transportation. In case of a medical emergency, the urban road is not free enough for an ambulance which may leads to a critical situation for the accident victim or the patient. By considering such scenarios, an ODIA model has been proposed which travels over air-transportation to the target area. Additionally, the proposed ODIA is driven by offline local maps and occupied with offline first-aid medical videos for helping the patients or victims located in a harsh and hostile environment. The ODIA is also occupied with online video streaming facility, but it only works when the internet connection properly available or else it works in the offline mode. This is the key strength of the ODIA model. Further, this work can be extended by the expansion of storage and battery capacity for long-distance coverage. 


\section{REFERENCES}

[1] K. T. Barkley, The Ambulance. Exposition Press, 1990.

[2] H. Mohapatra, et al., "Detection and avoidance of water loss through municipality taps in India by using smart taps and ICT," IET Wireless Sensor Systems, vol. 9, no. 6, pp. 447-457, 2019.

[3] H. Panda, H. Mohapatra, and A.K. Rath, "WSN-Based Water Channelization: An Approach of Smart Water," Smart Cities Opportunities and Challenges. Lecture Notes in Civil Engineering, vol 58, 2020.

[4] V. V. Krishna, et al., "Design of Drone Ambulance," International Journal of Pure and Applied Mathematics, vol. 119, no. 15, pp. 1813-1818, 2018.

[5] A. Momont, "The defibrillator drone that can beat ambulance times," https://www.bbc.com/news, 29 June 2017.

[6] A. Konert, J. Smereka, and L. Szarpak, "The Use of Drones in Emergency Medicine: Practical and Legal Aspects," Emergency Medicine International, vol. 2019, 2019, doi: 10.1155/2019/3589792.

[7] P. Van de Voorde, et al., "The drone ambulance [A-UAS]: golden bullet or just a blank?" Resuscitation, vol. 116, pp. 46-48, 2017.

[8] T. Amukele, et al., "Drone transportation of blood products," Transfusion, vol. 57, no. 3, pp. 582-588, 2017.

[9] D. Sachan, "The age of drones: what might it mean for health?" The Lancet, vol. 387, no. 10030, pp. 1803-1804, 2016.

[10] G. Van Berlaer, et al., "Disaster preparedness and response improvement: comparison of the 2010 Haiti earthquake-related diagnoses with baseline medical data," European Journal of Emergency Medicine, vol. 24, no. 5, pp. 382-388, 2017.

[11] Z. Dukowitz, "Drones in Search and Rescue: 5 Stories Showcasing Ways Search and Rescue Uses Drones to Save Lives," https://uavcoach.com/search-and-rescue-drones/, 18 January 2019.

[12] M. Balasingam, "Drones in medicinethe rise of the machines," International Journal of Clinical Practice, vol. 71, no. 9, 2017.

[13] J. B. Rosser, et al., "Medical applications of drones for disaster relief: a review of the literature," Surgical Technology International, vol. 33, pp. 17-22, 2018.

[14] J. Braun, et al., "The promising future of drones in prehospital medical care and its application to battlefield medicine," Journal of Trauma and Acute Care Surgery, vol. 87, no. 1S Suppl 1, pp. S28-S34, 2019.

[15] B. M. Bogle, et al., "The case for drone assisted emergency response to cardiac arrest: an optimized statewide deployment approach," North Carolina Medical Journal, vol. 80, no. 4, pp. 204-212, 2019.

[16] J. Scott and C. Scott, "Drone Delivery Models for Healthcare", in Hawaii International Conference on System Sciences 2017, 2017, doi: 10.24251/HICSS.2017.399.

[17] J. Lennartsson, "Strategic Placement of Ambulance Drones for Delivering Defibrillators to Out of Hospital Cardiac Arrest Victims," 2015.

[18] A. Goodchild, et al., "Delivery by drone: An evaluation of unmanned aerial vehicle technology in reducing CO2 emissions in the delivery service industry," Transportation Research Part D: Transport and Environment, vol. 61, pp. 58-67, 2018, doi: 10.1016/j.trd.2017.02.017.

[19] H. Mohapatra and A. K. Rath, "Fault tolerance in WSN through PE-LEACH protocol," IET Wireless Sensor Systems, vol. 9, no. 6, pp. 358-365, 122019.

[20] H. Mohapatra and A. K. Rath, "Fault-tolerant mechanism for wireless sensor network," IET Wireless Sensor Systems, vol. 10, no. 1, pp. 23-30, 22020.

[21] H. Mohapatra and A.K. Rath, "Fault Tolerance Through Energy Balanced Cluster Formation (EBCF) in WSN," in Smart Innovations in Communication and Computational Sciences. Advances in Intelligent Systems and Computing, S. Tiwari, et al., Eds., 2019.

[22] H. Panda, H. Mohapatra, and A.K. Rath, "WSN-Based Water Channelization: An Approach of Smart Water," in Smart CitiesOpportunities and Challenges. Lecture Notes in Civil Engineering, S. Ahmed, S. Abbas, H. Zia, Eds, 2020.

[23] M. Frank, "How Well Do Google Maps Work Offline? Absolutely Flawlessly," https://www.outsideonline.com/2411854/dog-shock-collar-training-experience, Nov 25, 2015.

[24] Blade Chroma RTF 4K HD Aerial Video Drone FPV GPS BLH8675 (Display Unit), https://www.worthpoint.com/worthopedia/blade-chroma-rtf-4k-hd-aerial-video-1811604589.

\section{BIOGRAPHY OF AUTHOR}

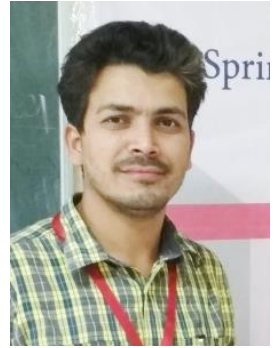

Hitesh Mohapatra received the Bachelor of Engineering degree in Information Technology from Biju Patnaik University of Technology (BPUT), Odisha in 2006, the masters in technology degree in CSE from Biju Patnaik University of Technology, Odisha in 2009. He is currently a full time Ph.D. scholar at Veer Surendra Sai University of Technology (VSSUT), Burla, India since 2017. He has contributed 20 SCI/Scopus level research papers, 8 international/national conferences and 2 books on Software Engineering and C Programming. He has 11 years of teaching experience both in industry and academia. His research interests include wireless sensor network, smart city, smart grid, and smart water. Further info on his pages: https://g.co/kgs/7F7qwd. 